\title{
Cellular scanning strategy for selective laser melting: Generating reliable, optimized scanning paths and processing parameters
}

\author{
Mohanty, Sankhya; Hattel, Jesper Henri
}

Published in:

Proceedings of SPIE

Link to article, DOI:

$10.1117 / 12.2079957$

Publication date:

2015

Document Version

Publisher's PDF, also known as Version of record

Link back to DTU Orbit

Citation (APA):

Mohanty, S., \& Hattel, J. H. (2015). Cellular scanning strategy for selective laser melting: Generating reliable, optimized scanning paths and processing parameters. In H. Helvajian, A. Piqué, M. Wegener, \& B. Gu (Eds.), Proceedings of SPIE: Laser 3D Manufacturing II (Vol. 9353). [93530U] SPIE - International Society for Optical Engineering. Proceedings of SPIE - The International Society for Optical Engineering https://doi.org/10.1117/12.2079957

\section{General rights}

Copyright and moral rights for the publications made accessible in the public portal are retained by the authors and/or other copyright owners and it is a condition of accessing publications that users recognise and abide by the legal requirements associated with these rights.

- Users may download and print one copy of any publication from the public portal for the purpose of private study or research.

- You may not further distribute the material or use it for any profit-making activity or commercial gain

- You may freely distribute the URL identifying the publication in the public portal 


\title{
Cellular scanning strategy for selective laser melting: Generating reliable, optimized scanning paths \& processing parameters
}

\author{
Sankhya Mohanty, Jesper Henri Hattel \\ Dept. of Mechanical Engineering, Technical University of Denmark, Produktionstorvet, \\ Kongens Lyngby, Denmark 2800
}

\begin{abstract}
Selective laser melting is yet to become a standardized industrial manufacturing technique. The process continues to suffer from defects such as distortions, residual stresses, localized deformations and warpage caused primarily due to the localized heating, rapid cooling and high temperature gradients that occur during the process. While process monitoring and control of selective laser melting is an active area of research, establishing the reliability and robustness of the process still remains a challenge.

In this paper, a methodology for generating reliable, optimized scanning paths and process parameters for selective laser melting of a standard sample is introduced. The processing of the sample is simulated by sequentially coupling a calibrated 3D pseudo-analytical thermal model with a 3D finite element mechanical model.

The optimized processing parameters are subjected to a Monte Carlo method based uncertainty and reliability analysis. The reliability of the scanning paths are established using cumulative probability distribution functions for process output criteria such as sample density, thermal homogeneity, etc. A customized genetic algorithm is used along with the simulation model to generate optimized cellular scanning strategies and processing parameters, with an objective of reducing thermal asymmetries and mechanical deformations. The optimized scanning strategies are used for selective laser melting of the standard samples, and experimental and numerical results are compared.
\end{abstract}

Keywords: SLM, scan strategies, optimized scan strategies , pseudo-analytical modeling, thermo-mechanical analysis, reliability estimation.

\section{INTRODUCTION}

The use of selective laser melting to make final products is increasing. While automotive and aerospace industries were the first to recognize the potential of additive rapid manufacturing, its usage is now spreading to smaller and mid-sized industries where it facilitates a platform for innovation. Thus, there is an increased effort towards developing procedures for in-process inspection and quality control including, but not limited to, thermal imaging [1] and ultrasonic [2] methods. Researchers have also attempted to establish reliability of the process by trying to characterize single scanning track formations using combination of statistical, numerical and experimental techniques [3] [4]. Simultaneously, optimization of the SLM process, particularly the scanning strategies, has also been an active area of research [5 $]^{[6]}$.

However, a proper framework for combining these various approaches, to enhance the process capabilities is still missing. In addition, researches involving the combined usage of experimental and numerical methods for characterizing SLM are still not as prevalent as in other laser manufacturing process. Thus, in this paper we try to propose such a framework which can be used to generate reliable, optimized scanning paths and processing parameters.

Laser 3D Manufacturing II, edited by Henry Helvajian, Alberto Piqué, Martin Wegener, Bo Gu Proc. of SPIE Vol. 9353, 93530U · C 2015 SPIE · CCC code: 0277-786X/15/\$18

doi: $10.1117 / 12.2079957$

Proc. of SPIE Vol. 9353 93530U-1 


\section{PHYSICAL MODEL DESCRIPTION}

\subsection{Thermal model}

Several thermal models for selective laser melting have been proposed in the recent years, each with their own assumptions and area of focus. In this study, the model proposed by Mohanty and Hattel for modelling SLM of single scanning tracks has been adopted [4]. The effect of powder bed properties and laser-material interaction has been taken into consideration, and modelled appropriately. While the model adopted is essentially a heat transfer model, the effects of mass transfer in the small melt pools associated with the process are captured indirectly by using equivalent material properties. Further, a pseudo-analytical method [7] of solving the governing thermal equations has been implemented which allows for significantly faster calculations with small loss in accuracy.

\subsection{Mechanical model}

As the process involves significantly high temperatures and melting-solidification, the actual physics governing the mechanical behavior is based on temperature-dependent plasticity. However, modelling any significantly large domain with the required complexity, usually results in large computation time. Researchers have tackled the problem by proposing that the effects on the mechanical behavior (e.g state of stress, strain and constitutive properties) by a particular layer only be considered after the whole layer has solidified [8].

Instead in this paper, a thermo-elastic model has been used with the understanding that while such models cannot predict accurate distribution of stresses/strains, they can nonetheless suggest the approximate levels of stress generated. The thermo-elastic model can especially predict where plastic yielding is likely to occur, and thereby regions in which larger residual stresses can be interpreted to occur. A further assumption is that of a continuum instead of particle based geometry. To achieve the appropriate constitutive behavior of powder layer, an artificially low value of thermal expansion coefficient and elastic modulus was implemented.

\section{THERMO-MECHANICAL MODELING OF SCANNING STRATEGY}

A 3D thermal pseudo-analytical model and a 3D mechanical model were developed to simulate different scan strategies for SLM. The pseudo-analytical model developed on MATLAB investigated the effects on the temperature field while producing a single layer using SLM. The mechanical modeling was carried out using the commercial software ABAQUS and took as input the temperature field predicted by the thermal model.

\subsection{Model description}

Figure 1 shows the typical model geometry and mesh. The physical domain used for simulation was $500 \mu \mathrm{m} X$ $500 \mu \mathrm{m}$ X $50 \mu \mathrm{m}$. In the pseudo-analytical model, the domain was divided into a structured mesh of $20 \times 20 \times 1$ elements (excluding ghost elements) having an element edge length of $25 \mu \mathrm{m}$ in (x,y) direction and $50 \mu \mathrm{m}$ in $\mathrm{z}$ direction. For the mechanical model, a structured mesh of 50 X 50 X 1 elements was generated with an element edge length of $10 \mu \mathrm{m}$ along the $\mathrm{XY}$ plane and $50 \mu \mathrm{m}$ along the z-direction. The temperature results from the pseudo-analytical model were interpolated and smoothed appropriately before being transferred to the mechanical model. 


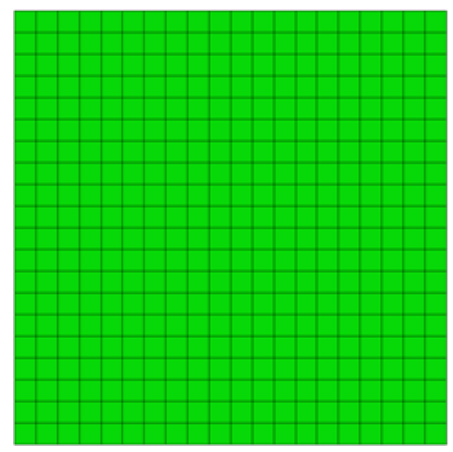

Figure 1 Problem domain floor simulation with structured mesh

\subsubsection{Problem specification}

As an example, the simulation of the parallel interlaced reverse scan path has been shown here. It is a modified version of parallel scan path (Figure 2d). The laser beam first scans the odd-numbered lines 1, 3, 5 and 7, as shown in figure, followed by the even-numbered lines 6,4 and 2 in that order.

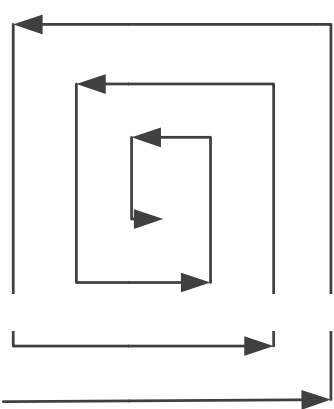

(a)

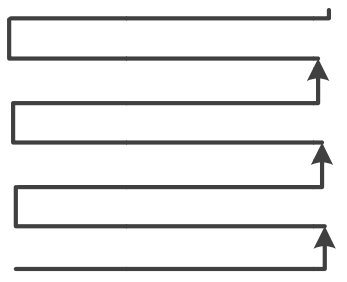

(c)

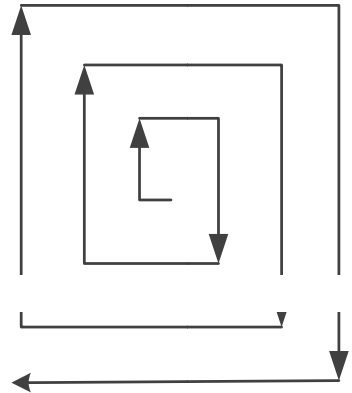

(b)

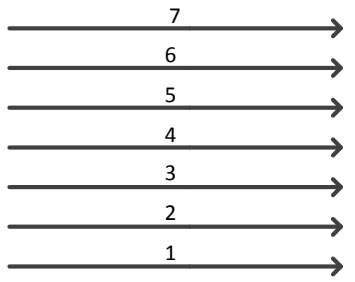

(d)

Figure 2 Scan strategies (a) In-spiral, (b) Out-spiral, (c) Antiparallel/Zigzag,

(d) Parallel, parallel-interlaced \& parallel-interlaced-reverse

\subsection{Results}

A typical output of interest from the simulation of the scan strategy is the temperature distribution in the domain (Figure 3 - bottom right). The temperature history of each element have been observed and based on the maximal temperature in the simulated processing time, elements have been categorized as molten or powder zones. As the mechanical properties for an element depend on whether it is powder or solidified-material, the temperature field has been tracked at each time step and the mechanical model follows the same time-step as the thermal model. The evolution of stress along the $\mathrm{x}$-axis (S11) and the y-axis (S22) have also been tracked and are shown in the top-left and top-left sections of Figure 3. A good 
indication of areas where plastic yielding would occur can be obtained by tracking the von Mises stress - a value higher than the yield strength of material would imply plastic deformation in such case. Thus, the von Mises stress has also been tracked and is shown in bottom-left section of Figure 3. Figure 4 and Figure 5 show the evolution of the temperature and stress fields as the scanning progresses.

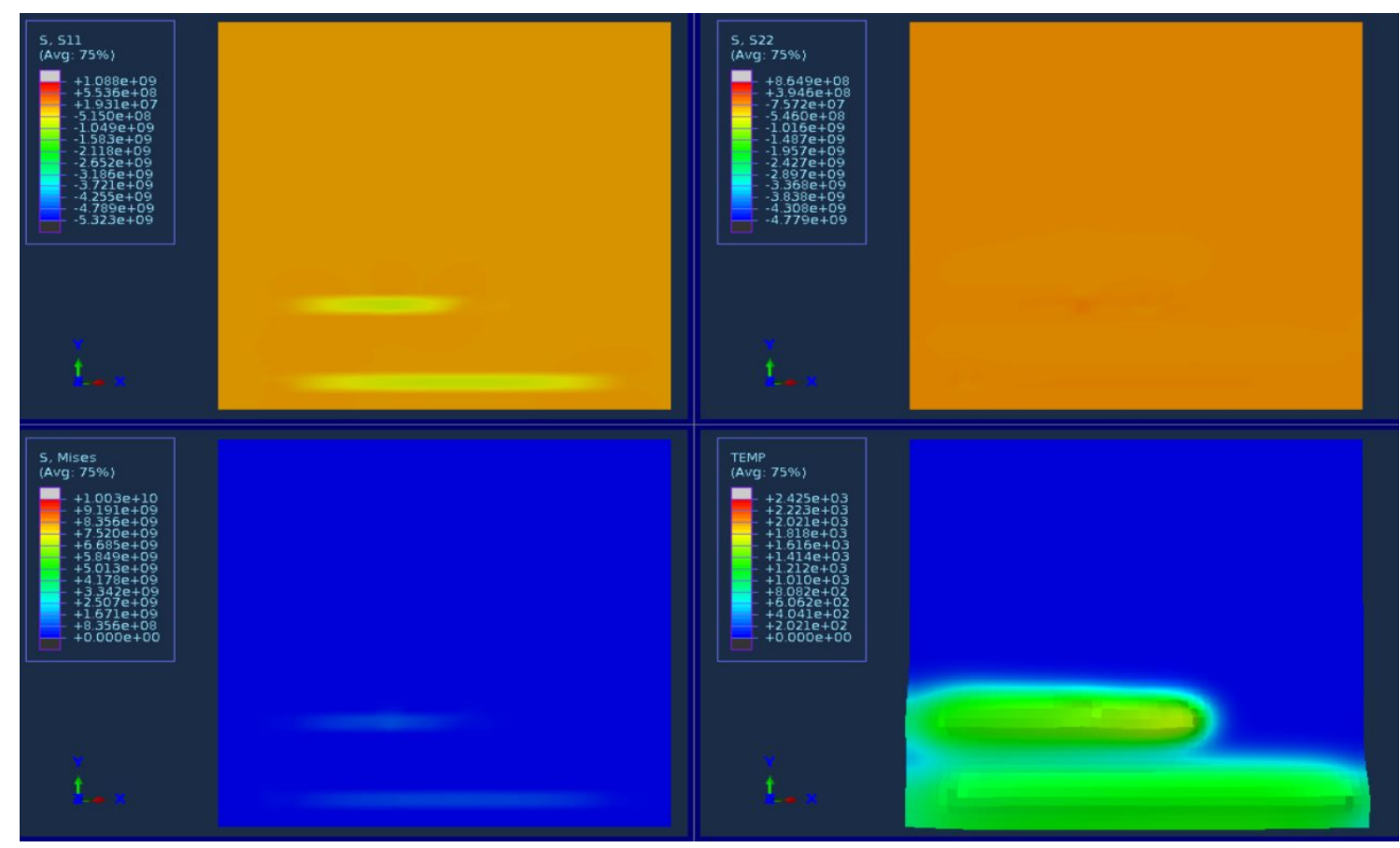

Figure 3 Temperature distribution, orthogonal normal stress along $\mathrm{x}$ - and $\mathrm{y}$-axis and von Mises stress fields during PIR scan path

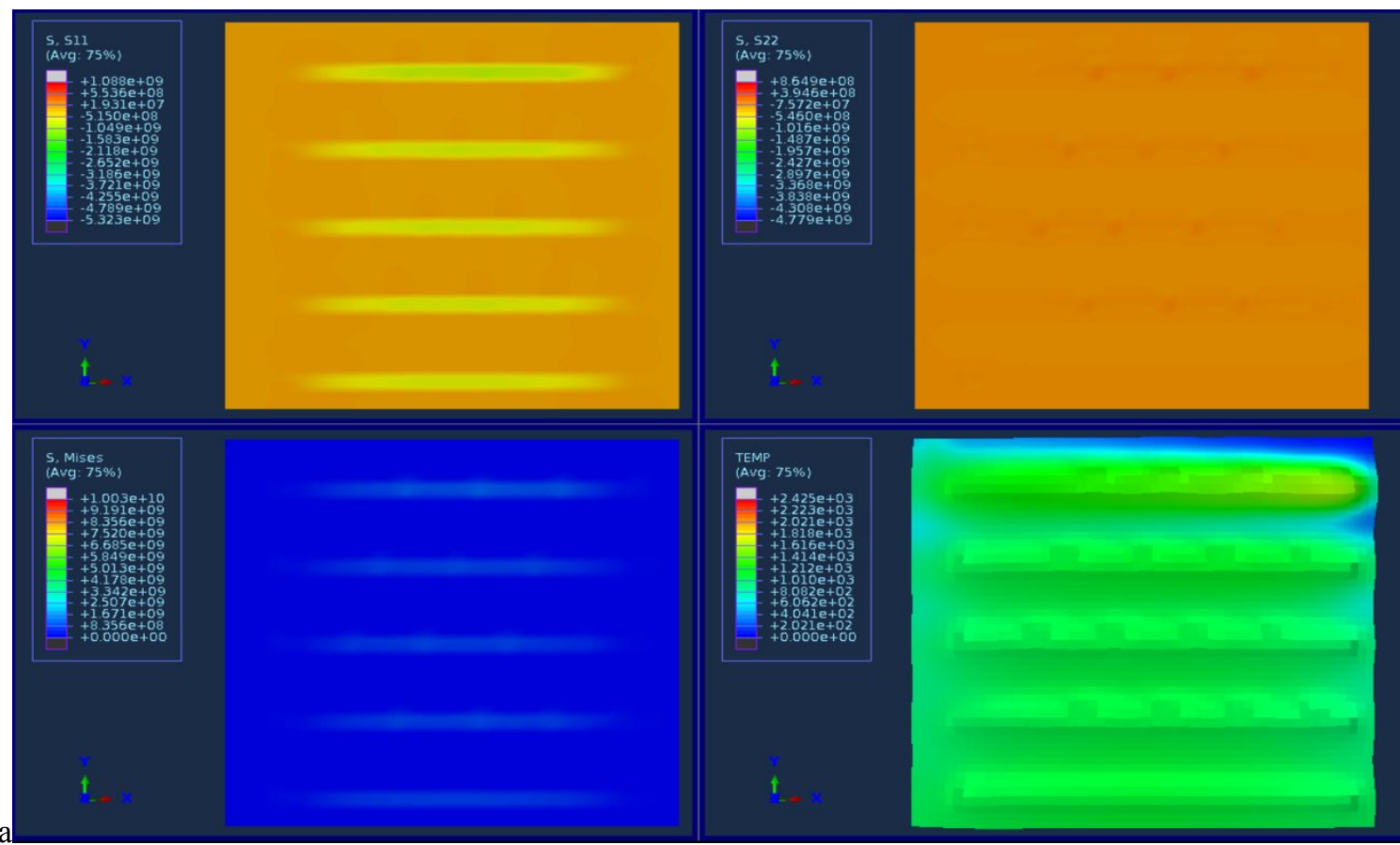

Figure 4 Temperature distribution, orthogonal normal stress along $\mathrm{x}$ - and $\mathrm{y}$-axis and von Mises stress fields during PIR scan path 


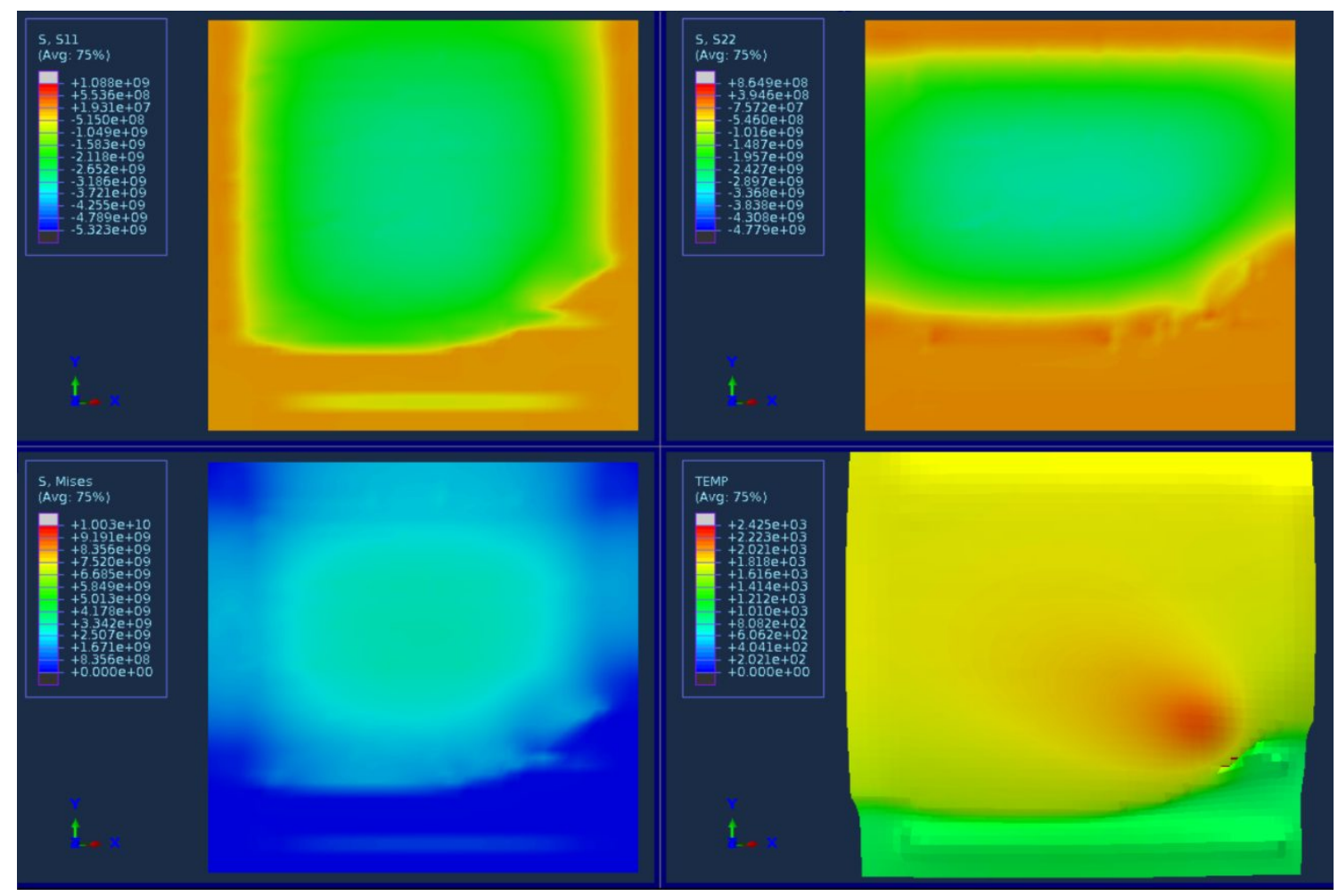

Figure 5 Temperature distribution, orthogonal normal stress along $\mathrm{x}$ - and $\mathrm{y}$-axis and von Mises stress fields during PIR scan path

It can be seen that initially the single tracks formed do not significantly influence each other. The heat conduction among them is not significant enough to create further melting of powder trapped between two tracks. Also, the tracks are not connected by solidified material and do not create any mechanical constraints for each other. The powder layer also does not pose as a constraining domain. Thus, only low levels of normal tensile stresses along x-axis are observed in the tracks, while those in y-axis are nearly non-existent.

However, during later part of the scan strategy, the existing tracks begin to experience partial remelting-resolidification and the increased conductivity significantly affects the temperature field. Moreover, existing tracks serve as physical constraints to newly formed tracks, and thus larger tensile and compressive stresses are observed along both axes. The von Mises stress is also seen to exceed the yield strength in the central region of the domain, thus suggesting that plastic yielding would occur at these locations. It can be observed that these high values of von Mises stresses correspond to areas which have undergone remelting-resolidification. It has also been found that the level of maximum von Mises stress has a positive correlation to the maximum temperature in the domain. However, it is necessary to consider that this is a typical observation in processes involving melting and rapid solidification, where the material suddenly goes from a state of zero/negligible strain (liquid) to having large tensile stresses.

\section{UNCERTAINTY ANALYSIS OF PARAMETERS}

\subsection{Problem Description}

Calibration studies on single melt tracks created with SLM by Mohanty and Hattel [4] suggest that small perturbations in the laser related parameters such as power, beam FWHM and the corresponding absorptivity of the material had a greater effect on the track dimensions than other parameters considered in the study. It is also a common knowledge that the temperature of the processing chamber affects the properties of the parts made by SLM. Thus, these four parameters 
have been chosen for an uncertainty analysis. The methodology used for the uncertainty analysis is the similar to that used by Mohanty and Hattel [4], and is thus not described here.

In this study, the six scanning strategies, as shown in Figure 2, were considered for simulations. An uncertainty analysis was performed for the different scanning strategies with the percentage of area consolidated, the thermal homogeneity (standard deviation of temperature) and the maximum temperature as the outputs.

\subsection{Results}

The nominal values of the parameters used for the uncertainty analysis and the associated expert uncertainty values are given in Table 1. It is further assumed that the parameters can take any value within the range of uncertainty with equal probability. The results from Latin Hypercube sampling of the parameters is shown in Figure 6.

Table 1 Nominal values of parameters and associated expert uncertainty values used for uncertainty analysis

\begin{tabular}{lll}
\hline Parameter & Nominal Values & Expert Uncertainty \\
\hline Power & $120 \mathrm{~W}$ & $5 \%$ \\
FWHM & $37.5 \mathrm{e}-6 \mathrm{~m}$ & $5 \%$ \\
Chamber Temperature & $27 \mathrm{C}$ & $5 \%$ \\
Powder Albedo & 0.9 & $5 \%$ \\
\hline
\end{tabular}

The 400 parameter sets generated by the sampling technique are then subjected to Monte Carlo simulations for each of the six scanning strategies. The obtained values of percentage of area consolidated, standard deviation of temperature and maximum domain temperature are then represented via cumulative probability distribution functions (CDF). The results for the six scanning strategies are shown in Figure 7 through Figure 12.

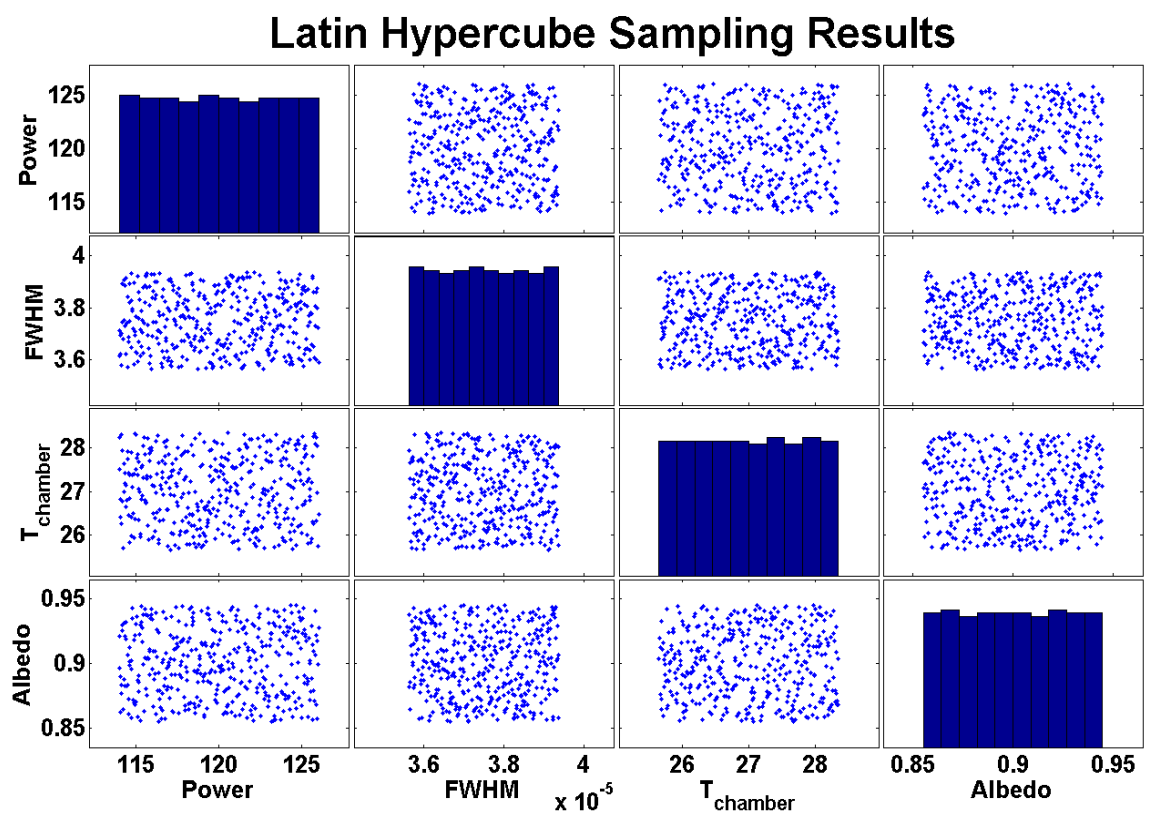

Figure 6 Latin Hypercube Sampling of parameter space for usage in uncertainty analysis 

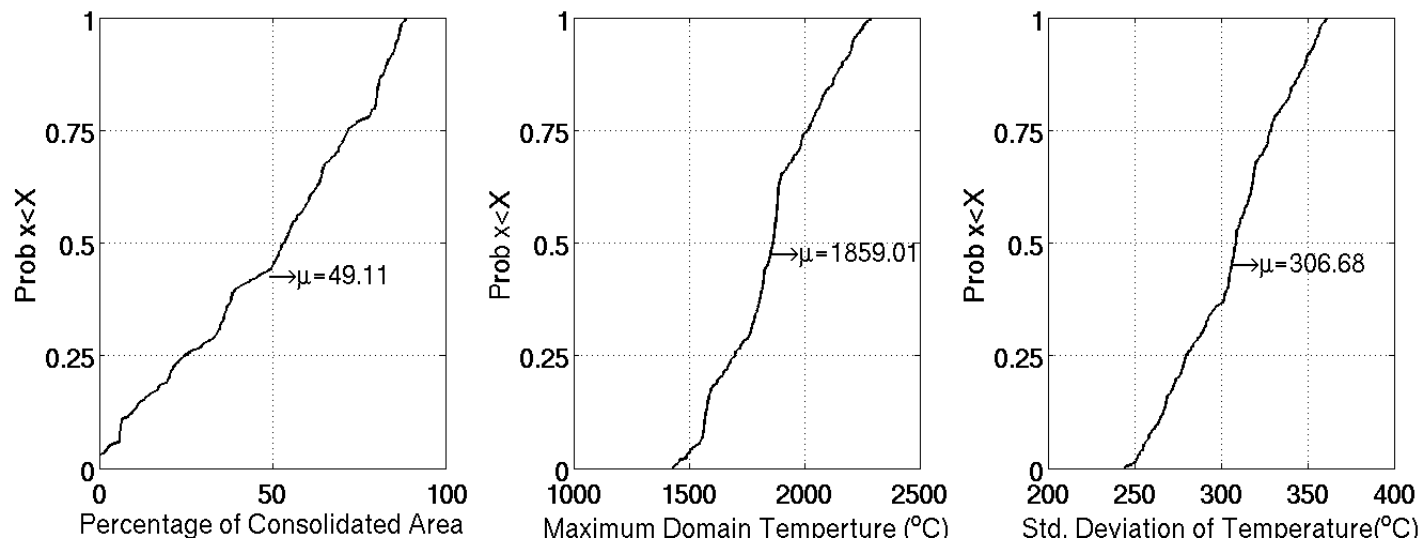

Figure 7 Cumulative probability distribution functions of percentage of consolidated area, maximum domain temperature and standard deviation of temperature for inspiral scanning path
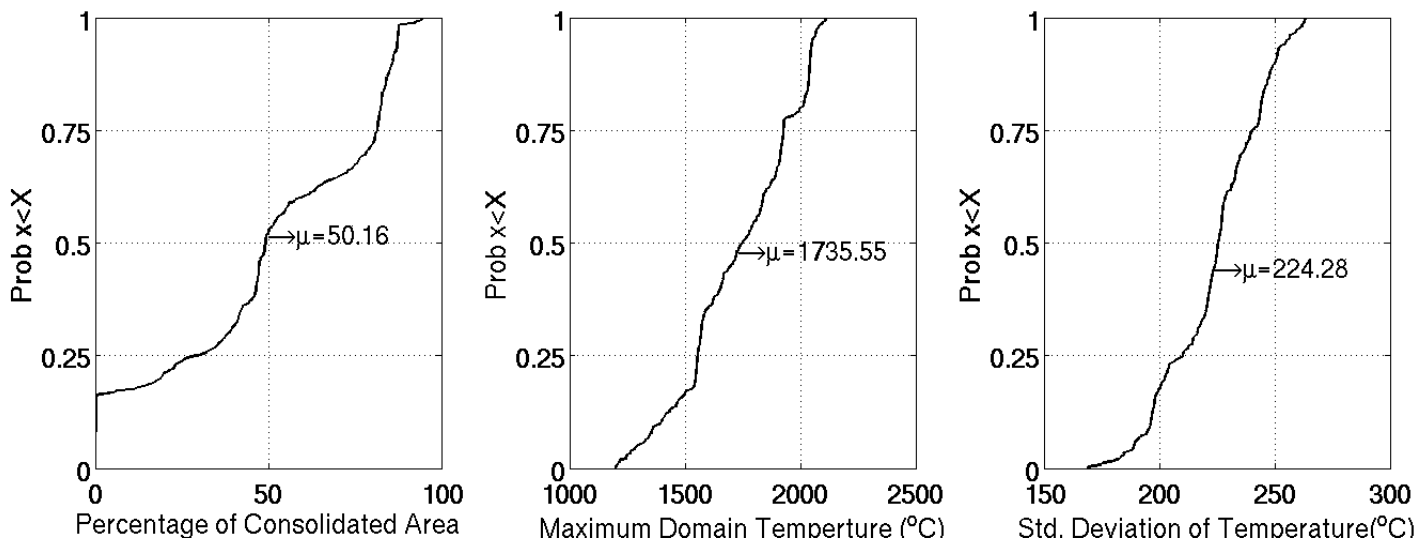

Figure 8 Cumulative probability distribution functions of percentage of consolidated area, maximum domain temperature and standard deviation of temperature for outspiral scanning path
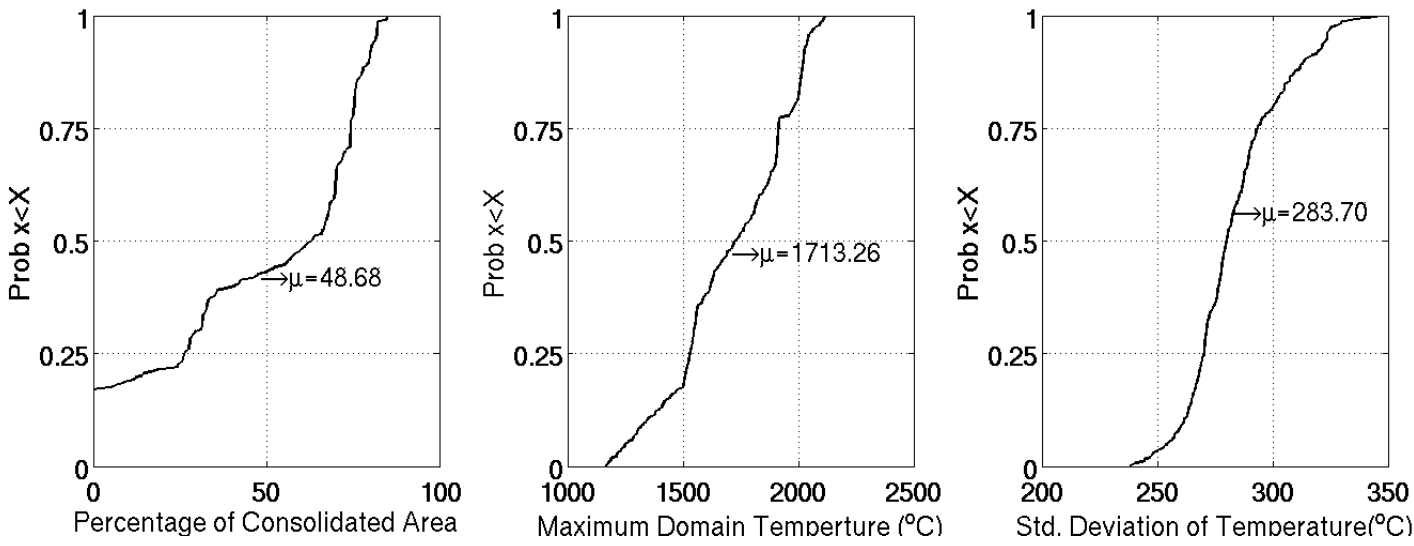

Figure 9 Cumulative probability distribution functions of percentage of consolidated area, maximum domain temperature and standard deviation of temperature for parallel scanning path 

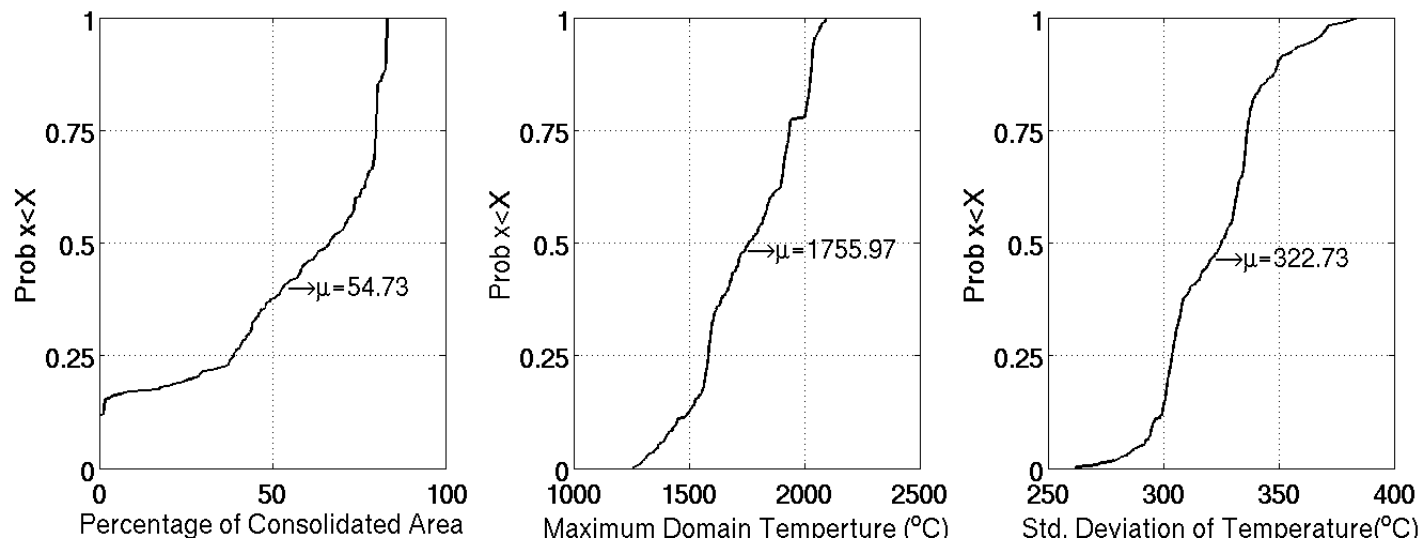

Figure 10 Cumulative probability distribution functions of percentage of consolidated area, maximum domain temperature and standard deviation of temperature for antiparallel/zigzag scanning path
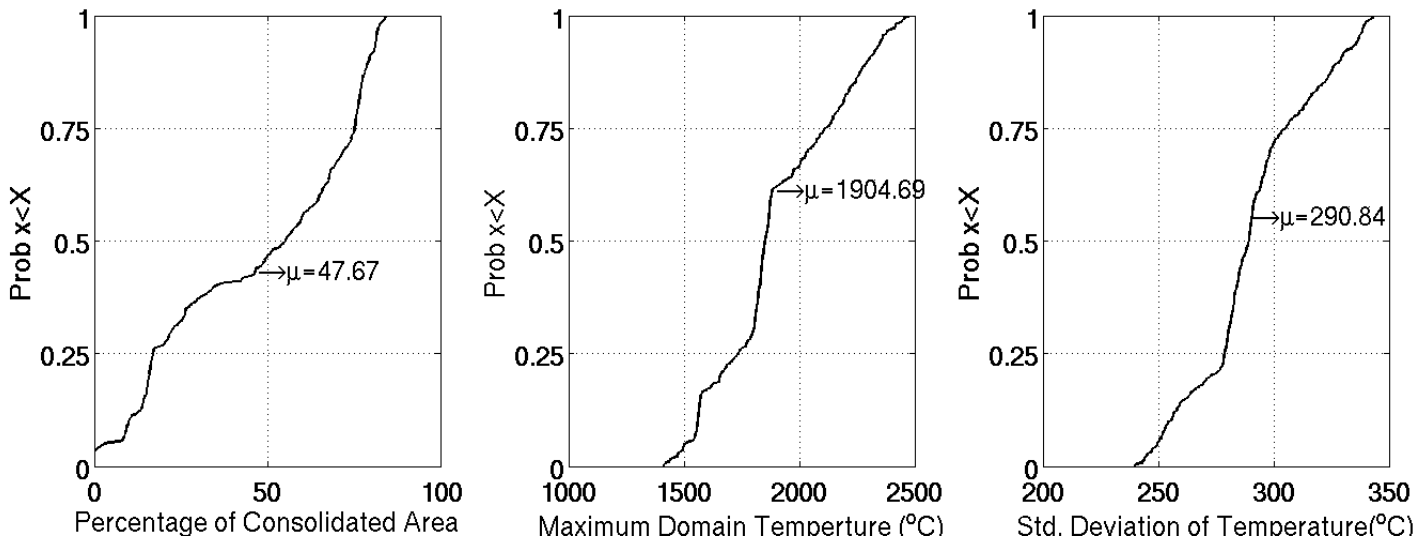

Figure 11 Cumulative probability distribution functions of percentage of consolidated area, maximum domain temperature and standard deviation of temperature for parallel interlaced scanning path
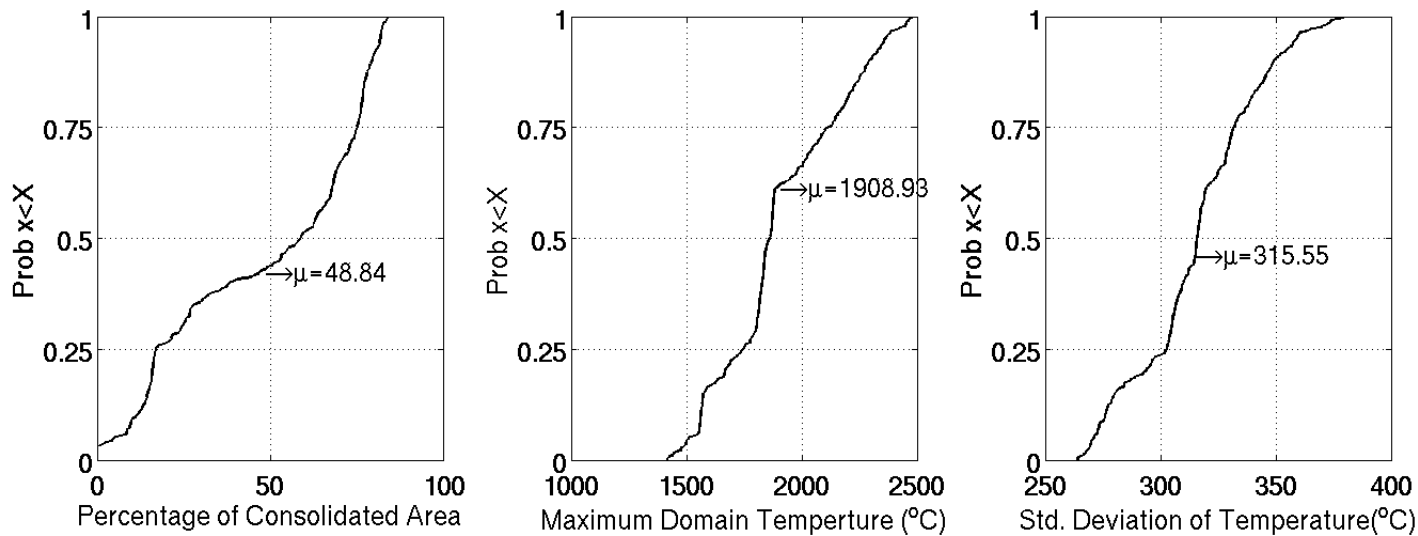

Figure 12 Cumulative probability distribution functions of percentage of consolidated area, maximum domain temperature and standard deviation of temperature for parallel interlaced reverse scanning path 
For each scanning strategy, the plots show the cumulative probability distribution for the percentage of consolidated area (left), maximum domain temperature(center) and the standard deviation of temperature at the end of scan path(right). As these are cumulative distribution curves, the steeper the slope of the curve, the more precise is the range of the corresponding output when the parameters can vary between $\pm 5 \%$ from their mean value. The plot also shows the average value of the percentage of consolidated area.

The average percentage of consolidated area for all the scanning paths is around $50 \pm 5 \%$. However, a gentler slope as seen for inspiral scanning strategy means that the scanning path is less robust. The steeper slopes seen three parallel scanning strategies imply the uncertainty in process parameters have less influence on the consolidated area. The plots however have a staircase-like appearance implying that the observed values are clustered around two ranges. Similar interpretations can also be extended to the two other outputs for the scanning paths. While the results do not show that any particular scan paths are exceedingly better than the other, they do hint at the robustness of each scan path.

\section{OPTIMIZATION OF SCANNING STRATEGIES}

After assessing the reliability of each scanning strategy, a study towards the optimization of the scanning pattern for selective laser melting process has been conducted. The default slicing softwares provided along with SLM machines typically divide each layer into multiple unit cells and then generate a processing sequence for these unit cells using some proprietary algorithms. From the perspective of thermal homogeneity (and thereby reduction of overall deformations), the processing sequence of these unit cells have significant influence. However, each unit cell is typically processed using parallel or antiparallel scanning strategies.

For the current optimization study, the modelled domain is increased to $1 \mathrm{~cm} \mathrm{X} 1 \mathrm{~cm} \mathrm{X} 50 \mu \mathrm{m}$. The square block is

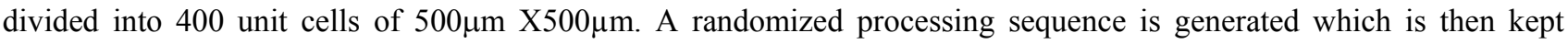
constant throughout the optimization study. As a reference case, each of these unit cells is processed by parallel scanning strategy.

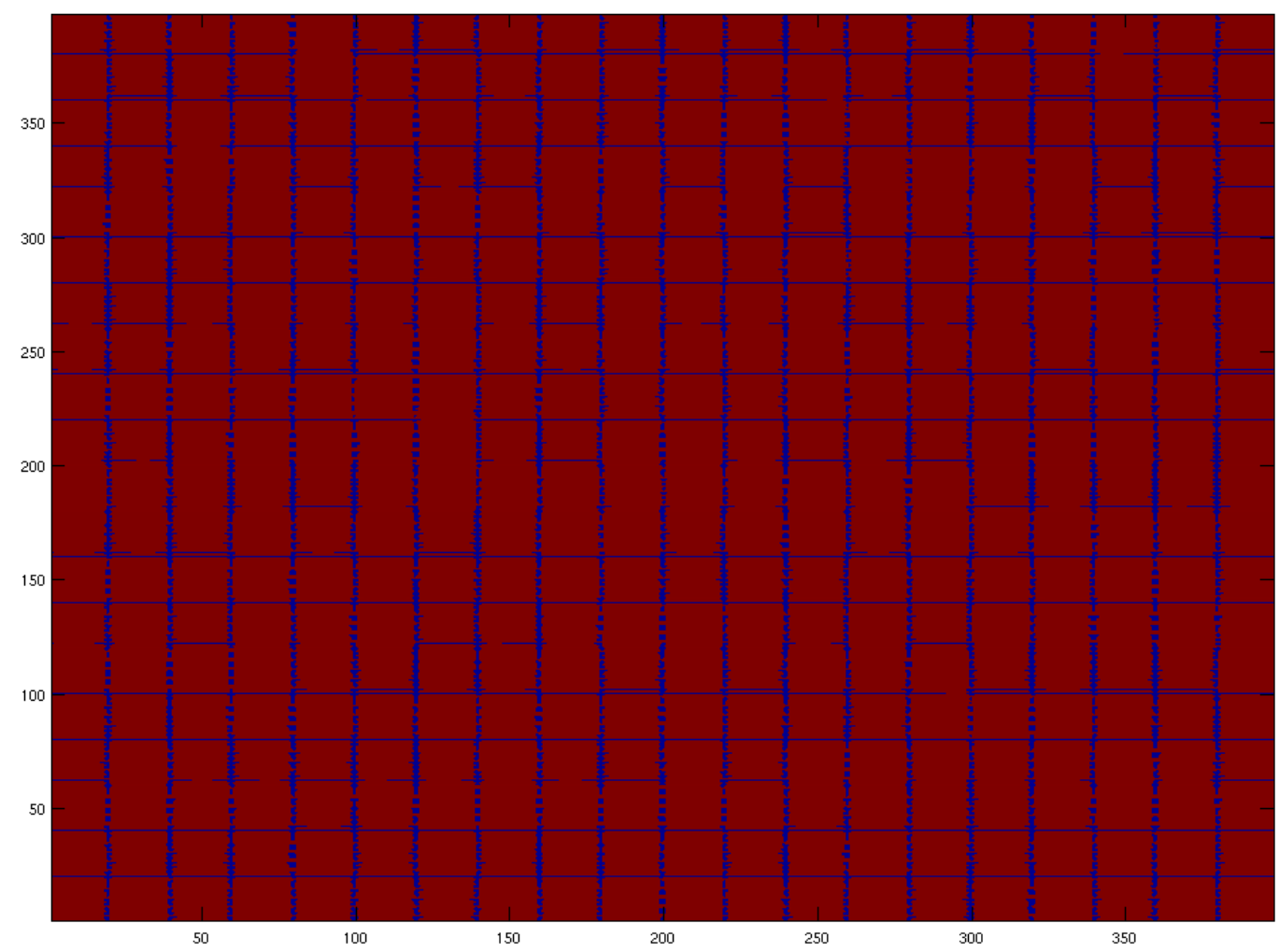

Figure 13 Area consolidated by processing each unit cell with parallel scanning strategy (Red=Consolidated, Blue=Powder) 
Figure 13 shows the area consolidated by processing each of the unit cells with the parallel scanning strategy. As can be observed, this results in a nice checkered board pattern of consolidation with unconsolidated powder mainly localized along the gridlines. In SLM machines, this is tackled by processing the gridlines either before or after the unit cells. However, the temperature field at the immediate end of the processing (as seen in Figure 14) contains several localized areas of high temperatures. While some degree of thermal homogeneity can be observed, the localized high temperature areas could lead to localized stress and/or deformations. The range of the color scheme for the figure has been defined to facilitate comparisons.

The same processing sequence is then used in a genetic algorithm based optimization performed to determine the appropriate scanning strategy for each unit cell, from within the six scan strategies. The dimensionless number $\theta$ (as defined by Mohanty and Hattel [7] ) for the whole domain is used as a fitness function for the optimization as it ensures a scanning strategy with greater thermal homogeneity, lower maximum domain temperature and greater consolidation.

The results for the $6^{\text {th }}$ best optimized scanning strategy are shown. As can be seen from Figure 15 , the unconsolidated area is not arranged as in the reference case. The temperature field shown in Figure 16, however, suggests better thermal homogeneity. The number of high temperature areas is reduced in comparison to the reference case, and the temperature in the domain is also reduced.

The optimized scanning strategies and the reference strategy are then used for producing single layers samples and cuboidal samples of 20 layer thickness on SLM machines. It is observed that the reference case begins to deform after addition of few layers, and rises above the powder layer, while the optimized scanning strategy has no such issues. However, the surface quality for the reference case is found to be better as a result of the arranged consolidation pattern.

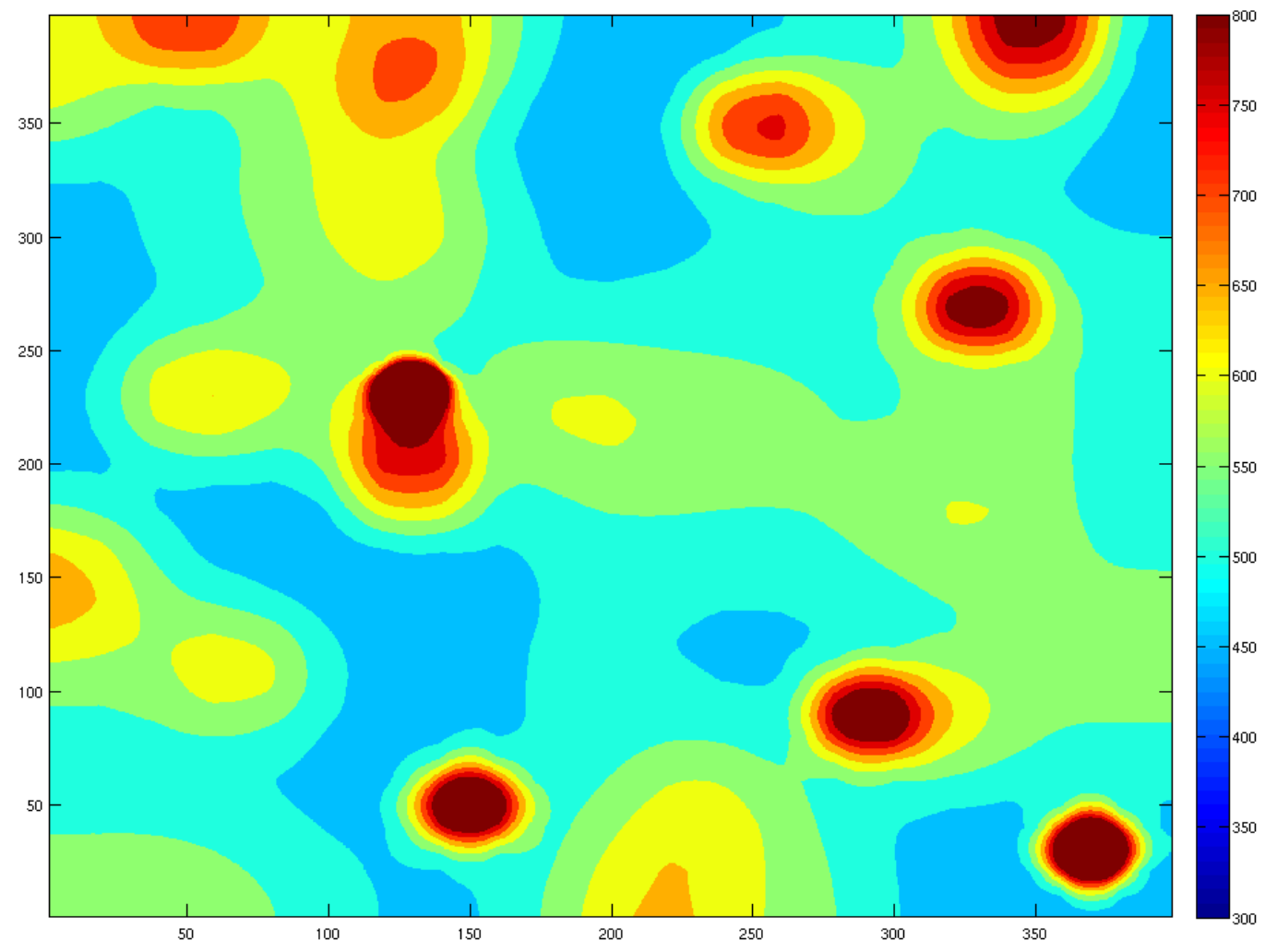

Figure 14 Temperature field at the end of processing each unit cell with parallel scanning strategy 


\section{DISCUSSIONS}

The current framework for generating reliable, optimized scanning strategies starts with considering different scanning paths for unit cells. The reliability of each scanning path with respect to the consolidation, maximal temperatures and thermal homogeneity has then been established. Only a limited set of parameters have been considered, however, and the reliability can be better established by considering more uncertainties in parameters such as powder bed characteristics and temperature-dependent material properties.

Next, these characterized unit cell scanning paths are used for optimization of cellular scanning strategy for standard sample. In the current study, most of the scanning paths used have preference along the $\mathrm{x}$-axis. It would be advisable to also consider rotated versions of these scan paths, in addition to other unit cell scan paths, to provide greater searching space for optimization algorithms. Also, the optimization of the processing sequence should be performed as it has a greater influence on the thermal homogeneity.

A more desirable framework would involve optimization of cellular scanning strategy using a fully-coupled thermomechanical model, followed by reliability estimation of the optimized scanning strategy by Monte Carlo methods. However, due to the large computation time associated with each model simulation such a framework cannot provide results within acceptable times.

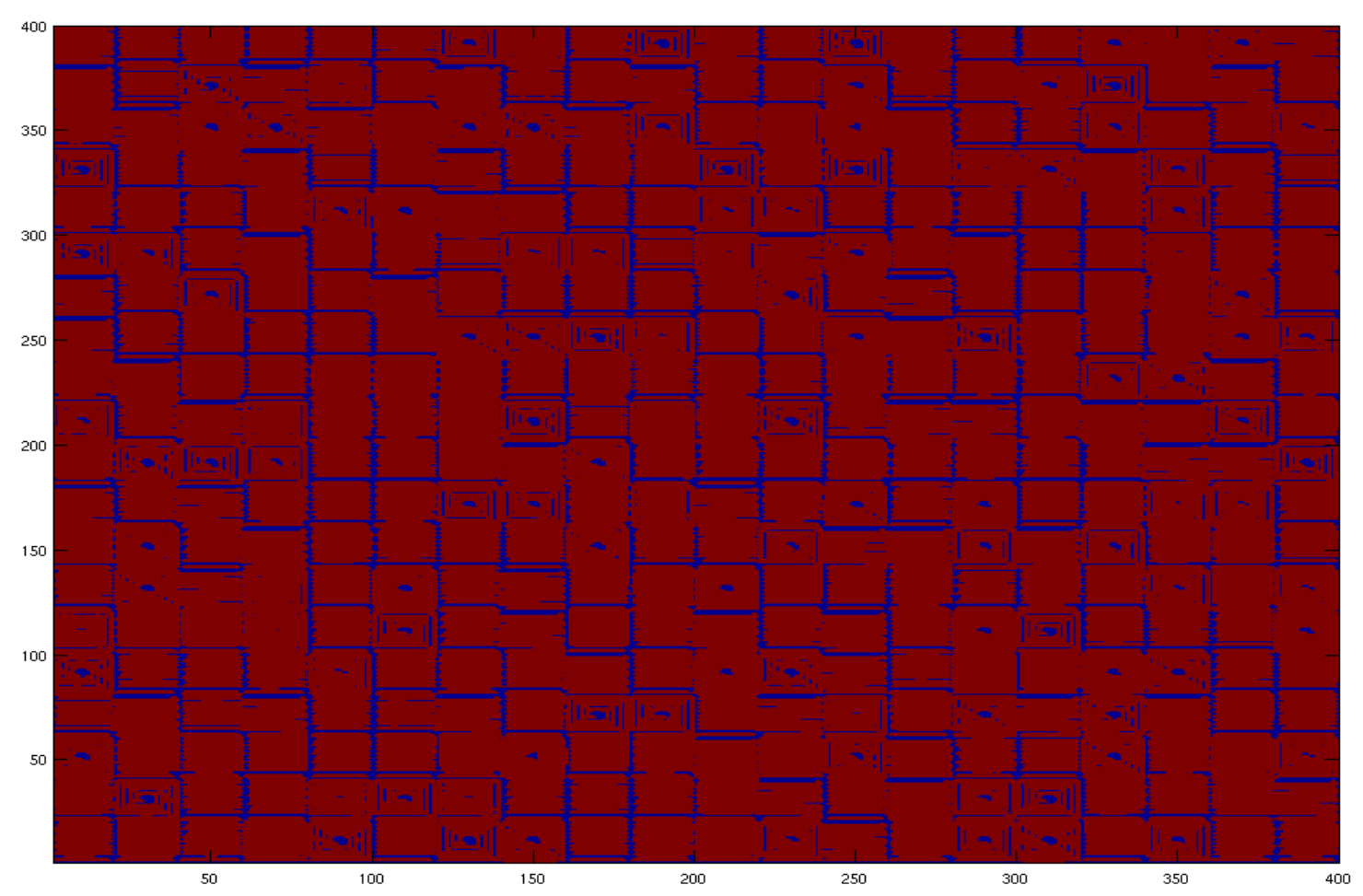

Figure 15 Area consolidated by optimized scanning strategy (Red=Consolidated, Blue=Powder) 


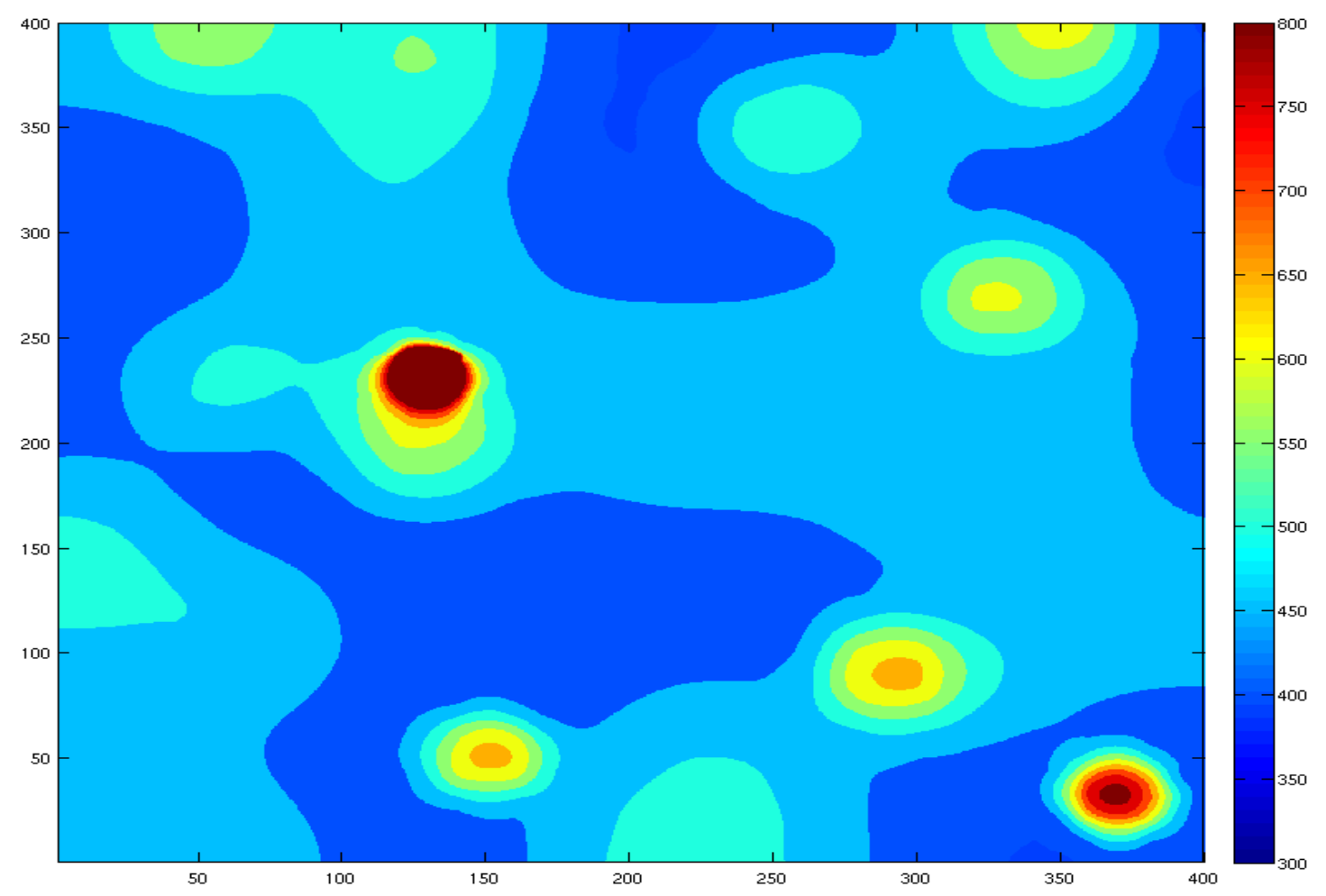

Figure 16 Temperature field at the end of optimized scanning strategy

\section{CONCLUSION}

A framework for generating reliable, optimized cellular scanning strategy for selective laser melting was proposed. Using appropriate thermal and mechanical models, the unit cell scan paths were simulated. Using Monte Carlo techniques, the reliability of these scan paths for the chosen parameters, with respect to select outputs, was determined. The unit cell scan paths were then used for optimization of a larger standard sample. Both numerical and experimental results show the optimized scan strategy to perform better than the reference case (with the criteria considered during optimization). Possible improvements to the framework were then discussed, which would be implemented in successive works as and when computational capabilities improve.

\section{REFERENCES}

[1] S. Clijsters, T. Craeghs, S. Buls, K. Kempen and J. Kruth, "In situ quality control of the selective laser melting process using a high speed, real-time melt pool monitoring system," The International Journal of Advanced Manufacturing Technology, vol. 75, no. 5-8, pp. 1089-1101, 2014.

[2] H. Rieder, A. DillHofer, M. Spies, J. Bamberg and T. Hess, "Online monitoring of additive manufacturing processes using ultrasound," in ECNDT 2014, Prague, 2014.

[3] I. Yadroitsev and I. Smurov, "Factor analysis of selective laser melting process parameters and geometrical characteristics of synthesized single tracks," Rapid Prototyping Journal, pp. 201-208, 2012.

[4] S. Mohanty and J. Hattel, "Numerical Model based Reliability Estimation of Selective Laser Melting Process," Physics Proceedia, vol. 56, pp. 379-389, 2014.

[5] R. Mertens, S. Clijsters, K. Kempen and J. Kruth, "Optimization of Scan Strategies in Selective Laser Melting of Aluminum Parts With Downfacing Areas," J. Manuf. Sci. Eng., vol. 136, no. 6, 2014. 
[6] S. Mohanty, C. Tutum and J. Hattel, "Cellular scanning strategy for selective laser melting: evolution of optimal grid-based scanning path and parametric approach to thermal homogeneity," in Proceedings of SPIE 8608, Laser-based Micro- and Nano-packaging and Assembly VII, San Francisco, USA, 2013.

[7] S. Mohanty and J. Hattel, "Cellular Scanning Strategy for Selective Laser Melting: Capturing Thermal Trends with a Low-Fidelity, Pseudo-Analytical Model," Mathematical Problems in Engineering, vol. 2014, no. Article ID 715058, p. 14 pages, 2014.

[8] M. Zaeh and B. G., "Investigations on residual stresses and deformations in selective laser melting," Production Engineering, vol. 4, no. 1, pp. 35-45, 2010. 